\title{
Interplay between electromagnetically induced transparency and Autler-Townes effect in five- level atomic systems
}

\author{
Gul Tiaz ${ }^{1}$, Fazal Ghafoor ${ }^{1, *}$, Rashid Nazmitdinov ${ }^{2,3}$, and Ehtiram Shahalyev ${ }^{4,5}$ \\ ${ }^{1}$ Department of Physics, COMSATS University, 44000 Islamabad, Pakistan \\ ${ }^{2}$ BLTP, Joint Institute for Nuclear Research, Dubna, Russia \\ ${ }^{3}$ Dubna State University, Dubna, Russia \\ ${ }^{4}$ VBLHEP, Joint Institute for Nuclear Research, Dubna, Russia \\ ${ }^{5}$ National Nuclear Research Center, Baku, Azerbaijan
}

\begin{abstract}
We analyse the evolution of a weak probe optical field propagation through a five-level atomic medium cyclically driven by two strong optical and microwave fields. It is shown that the competition between the electromagnetically induced transparency and the Autler-Townes effect can be controlled by altering the relative phase of the coupling fields in the presence of the atomic dephasing reservoir.
\end{abstract}

\section{Introduction}

Asymmetric Fano resonance, discussed in a seminal work by Ugo Fano [1], provides a remarkable concept, employed in various fields of physics nowadays. At its base is the interference effect between indistinguishable probability amplitudes of bound-to-continuum and, through auto-ionizing channel, the indirect bound-to-continuum transitions of ionized atom [1-5]. This concept helps to understand numerous interesting optical phenomena such as coherent population trapping [6], lasing without inversion [7], optically controlled slowing of light [8] and optical storage [9]. All these phenomena are the manifestation of the electromagnetically induced transparency (EIT) that is closely related to the Fano resonance [10].

The long-lived coherence in alkali metals and their simple electronics level structure made it possible to demonstrate the EIT experimentally. It has been investigated as well in quantum dots [11], nanoplasmonics [12, 13], superconducting circuits [14], metamaterials [15, 16], optomechanics [17], and inductively (capacitively) coupled electrical resonator circuits [18, 19]. Sensitive to the dynamical parameters [8], these coherence processes of the Fano-like interference effect may be more efficiently if the systems are assisted by the coherence due to multiple mode laser field coupling and interference [20].

New practical applications of the coherent processes may be revealed if the EIT with timescale separations in alkali metals based systems [21] could be demonstrated easily. However, the inherited decoherence limits these useful applications in such systems. The transparency, without the Fano-like interference, could also occur by means of the Autler-Townes effect

\footnotetext{
*e-mail: rishteen@yahoo.com
} 


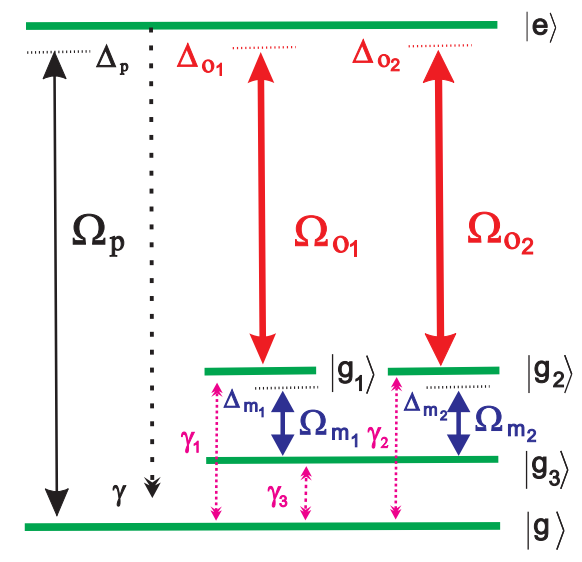

Figure 1. (Color online) Schematics of the atomic system

(ATE) [22-25], that was discussed in context of a doublet structure of an absorption profile of a probe light.

Based on the dressed state picture, Harris and Imamoglu [26] have shown the condition for the Fano-like interference in a $\Lambda$-type system. To discern the EIT from the AT effect, the approach of decaying dressed states was introduced by decomposing the response of strongly driven atomic system as a simple superposition of two resonances [27]. In addition, the threshold of the coupling strength could also discern the EIT from the ATE [28].

In order to elucidate the difference of the EIT from the ATE in the experiment involving a three-level atom, Sanders and co-workers made an objective test on empirical data [29]. The discerning the EIT from the ATE has been demonstrated experimentally in a cold cesium atomic system as well [30]. Moreover, Zhu and co-workers investigated the crossover from the EIT to the ATE in the open V-type hot molecular system [31].

The purpose of the present contribution is to trace the interplay between the EIT and the ATE in a five-level atomic system. In particular, we investigate the coherence evolution of a weak optical field probed through the atomic system. The atomic system is driven by two strong optical and microwave fields cyclically in the presence of an atomic dephasing rate in the medium. The structure of the contribution is the following. In Sec.2, we describe the model; the calculation of the response function of the probe field, propagation through the medium. The numerical results of our analysis are presented in Sec.3. The main conclusions are summarized in Sec. 4.

\section{Coherence evolution of a five-level atomic medium}

\subsection{The Model}

We analyse a five-level atomic system driven cyclically by two strong microwave and two strong optical fields in the presence of the weak probe field (see Fig. 1). The energy level $|e\rangle$ is coupled to the energy levels $\left|g_{1}\right\rangle$ and $\left|g_{2}\right\rangle$ via two strong optical fields of frequencies $\omega_{O 1}$ and $\omega_{O 2}$ with the Rabi frequencies $\Omega_{O 1}, \Omega_{O 2}$ (creating a $\Lambda$-type configuration). On the other hand, the levels $\left|g_{1}\right\rangle$ and $\left|g_{2}\right\rangle$ are coupled to the energy level $\left|g_{3}\right\rangle$ via two microwave fields of carrier frequencies $\omega_{m 1}$ and $\omega_{m 2}$ with the Rabi frequencies $\Omega_{m 1}, \Omega_{m 2}$, respectively (a V-type configuration). In order to study the transmission behaviour through the medium, we 
introduce the optical weak probe field of frequency $\omega_{p}$. It is coupled to the energy level $|e\rangle$, and $|g\rangle$ with the Rabi frequency $\Omega_{p}$. We consider the radiative decay rate $\gamma$ from the excited $|e\rangle$ to the lower energy level $|g\rangle$ and neglect the decay rates to the lower energy levels $\left|g_{1}\right\rangle$, $\left|g_{2}\right\rangle$, and $\left|g_{3}\right\rangle$, in the presence of the strong coupling fields along the corresponding atomic transitions.

Thus, in the interaction picture, using the rotating wave and electric dipole approximations, we analyse the following Hamiltonian

$$
\begin{aligned}
H_{I} & =-\frac{\hbar}{2}\left[\Omega_{m 1} \exp \left[i\left(\Delta_{m 1} t+\phi_{m 1}\right)\right]\left|g_{1}\right\rangle\left\langle g_{3}\left|+\Omega_{m 2} \exp \left[i\left(\Delta_{m 2} t+\phi_{m 2}\right)\right]\right| g_{2}\right\rangle\left\langle g_{3}\right|\right. \\
& +\Omega_{O 1} \exp \left[i\left(\Delta_{O 1} t+\phi_{O 1}\right)\right]|e\rangle\left\langle g_{1}\right|+\Omega_{O 2} \exp \left[i\left(\Delta_{O 2} t+\phi_{O 2}\right)|e\rangle\left\langle g_{2}\right|\right] \\
& \left.+\Omega_{p} \exp \left[i\left(\Delta_{p} t+\phi_{p}\right)\right]|e\rangle\langle g|\right]+ \text { H.c. },
\end{aligned}
$$

where $\Delta_{O 1(O 2)}=\omega_{e g_{1(2)}}-\omega_{O 1(O 2)}$ is the detuning of the optical field with the frequency $\omega_{O 1}$ $\left(\omega_{O 2}\right)$. The detuning of the microwave with the frequency $\omega_{m 1}\left(\omega_{m 2},\right)$ is defined as $\Delta_{m 1}=$ $\omega_{g_{1} g_{3}}-\omega_{m 1}\left(\Delta_{m 2}=\omega_{g_{2} g_{3}}-\omega_{m 2}\right)$. The detuning of the probe field with the carrier frequency $\omega_{p}$ is denoted as $\Delta_{p}=\omega_{e g}-\omega_{p}$.

In the steady state limit, the transition density matrix element of the probe field is defined by the master equation of motion

$$
\dot{\rho}=-\frac{i}{\hbar}\left[H_{I}, \rho\right]-\frac{1}{2} \Gamma \sum\left(\sigma^{\dagger} \sigma \rho+\rho \sigma^{\dagger} \sigma-2 \sigma \rho \sigma^{\dagger}\right),
$$

where, $\sigma$ and $\sigma^{\dagger}$ are the lowering and raising operators associated with the atomic radiative and dephasing decay rates in the atomic medium, which we consider to be loaded in a vapor cell.

\subsection{Response function of the medium}

The response function of the system is considered up to the first order with respect to the weak probe field, and up to all orders with respect to the two strong optical and microwave fields. In this approximation, the real and imaginary parts of the transition matrix element $\rho_{e g}$ is defined as [32]

$$
\begin{aligned}
\operatorname{Re}\left[\rho_{e g}\right]= & \frac{2 \Omega_{p}}{\left(D_{1}^{2}+D_{2}^{2}\right)}\left[D_{2}\left\{4 \gamma_{1} A-4 S_{11} B+\gamma_{1} \Omega_{m 2}^{2}+\gamma_{2} \Omega_{m 1}^{2}\right\}\right. \\
& \left.-D_{1}\left\{4 \gamma_{1} B+4 S_{11} A+\Omega_{m 2}^{2} S_{11}+\Omega_{m 1}^{2} S_{22}\right\}\right], \\
\operatorname{Im}\left[\rho_{e g}\right]= & \frac{2 \Omega_{p}}{\left(D_{1}^{2}+D_{2}^{2}\right)}\left[D_{1}\left\{4 \gamma_{1} A-4 S_{11} B+\gamma_{1} \Omega_{m 2}^{2}+\gamma_{2} \Omega_{m 1}^{2}\right\}\right. \\
& \left.+D_{2}\left\{4 \gamma_{1} B+4 S_{11} A+\Omega_{m 2}^{2} S_{11}+\Omega_{m 1}^{2} S_{22}\right\}\right],
\end{aligned}
$$

where

$$
\begin{aligned}
D_{1}= & 16(E A-F B)+4\left\{\Omega_{m 2}^{2} E+\Omega_{m 1}^{2} C+\Omega_{O 1}^{2} A\right. \\
& \left.+\Omega_{O 2}^{2}\left(\gamma_{1} \gamma_{3}-S_{11} S_{33}\right)\right\}+\Omega_{O 1}^{2} \Omega_{m 2}^{2}+\Omega_{O 2}^{2} \Omega_{m 1}^{2}-\Omega_{e f f}^{4} \cos [\phi], \\
D_{2}= & 16(E B+F A)+4\left\{\Omega_{m 2}^{2} F+\Omega_{m 1}^{2} D+\Omega_{O 1}^{2} B+\left(\gamma_{1} S_{33}+\gamma_{3} S_{11}\right) \Omega_{O 2}^{2}\right\}, \\
A= & \left(\gamma_{2} \gamma_{3}-S_{22} S_{33}\right), B=\left(\gamma_{2} S_{33}+\gamma_{3} S_{22}\right), C=\left(\gamma \gamma_{2}-\Delta_{p} S_{22}\right), \\
D= & \left(\gamma S_{22}+\gamma_{2} \Delta_{p}\right), E=\left(\gamma \gamma_{1}-\Delta_{p} S_{11}\right), F=\left(\gamma S_{11}+\gamma_{1} \Delta_{p}\right), \\
\Omega_{e f f}^{4}= & 2 \Omega_{O 1} \Omega_{O 2} \Omega_{m 1} \Omega_{m 2}, \phi=\phi_{O 1}+\phi_{m 1}-\left(\phi_{O 2}+\phi_{m 2}\right) .
\end{aligned}
$$




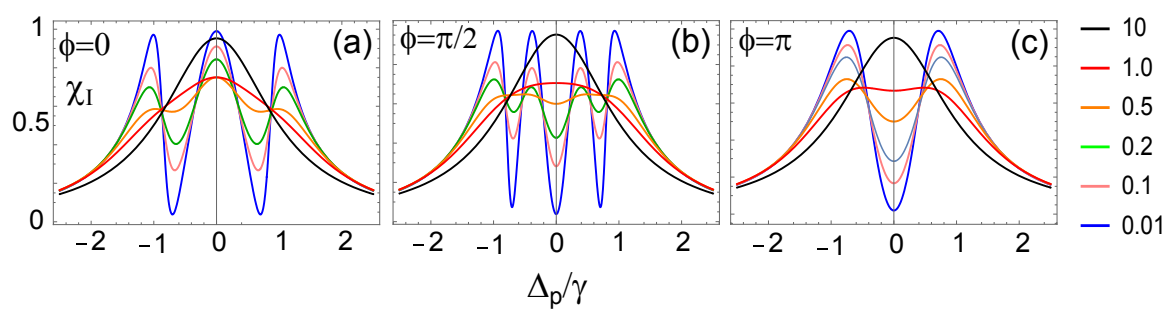

Figure 2. (Color online) Absorption $\operatorname{Im} \chi\left(\Delta_{p}\right)$ versus $\Delta_{p} / \gamma$ is shown with $\Omega_{O 1}=\Omega_{O 2}=\Omega_{m 1}=\Omega_{m 2}=$ $1 \gamma$ and with (a) $\phi=0$ (triplet), (b) $\phi=\pi / 2$ (quartuplet) and (c) $\phi=\pi$ (doublet). The dephasing decay rates $\gamma_{i=1,2,3}$ are selected as $0.01 \gamma$ (blue), $0.1 \gamma$ (pink), $0.2 \gamma$ (green), $0.5 \gamma$ (orange), $1.0 \gamma$ (red), $10 \gamma$ (black). The behaviour of the system with the dephasing rates $0.01 \gamma$ and $0.5 \gamma$ are typical examples of the EIT and the ATE in each case of the relative phase $\phi$, respectively

Here, $S_{11}=\left(\Delta_{p}-\Delta_{O 1}\right), S_{22}=\left(\Delta_{p}-\Delta_{O 2}\right)$ and $S_{33}=\left(\Delta_{p}-\Delta_{O 2}-\Delta_{m 2}\right)$. The absorption and dispersion of the probe field are investigating with the aid of the imaginary and real parts of the electric susceptibility of the atomic system:

$$
\begin{aligned}
& \operatorname{Im}\left[\chi\left(\Delta_{p}\right)\right]=\frac{2 N\left|\wp_{e g}\right|^{2}}{\epsilon_{0} \hbar \Omega_{p}} \operatorname{Im}\left[\rho_{e g}\right], \\
& \operatorname{Re}\left[\chi\left(\Delta_{p}\right)\right]=\frac{2 N\left|\wp_{e g}\right|^{2}}{\epsilon_{0} \hbar \Omega_{p}} \operatorname{Re}\left[\rho_{e g}\right],
\end{aligned}
$$

respectively. Here, $\wp_{e g}$ is the matrix element of the electric dipole moment, $N$ is the atomic number density, and $\rho_{e g}$ is the density matrix element of the atomic transition between states $|e\rangle$ and $|g\rangle$. While considering the EIT in the linear theory regime, the Rabi frequency of the probe field, with the atomic dipole matrix element $\wp_{e g}$, is dealt in the regime of weak atom-field coupling $\Omega_{p}=0.01 \gamma$.

\section{Results and discussion}

\subsection{Coherent evolution of the triple EIT}

First, we analyse a simple case. The atomic medium is considered to be cold, i.e., in the BoseEinstein condensate state (BEC). The atomic velocity is expected to be very small. Thereby, the dephasing decay rates caused by the atomic collisions in the medium may be neglected safely. In this case, we use $\Delta_{O 1}=\Delta_{O 2}=\Delta_{m 1}=\Delta_{m 2}=0$, and $\gamma_{i} \approx 0(i=1-3)$ in the analysis of Eq. (6). The positions of the dark lines (under these conditions) are determined by the roots of $\Delta_{p}$, when the numerator of Eq. (6) is zero. Consequently, two of them are located at

$$
\Delta_{p}= \pm \frac{1}{2} \sqrt{\Omega_{m 1}^{2}+\Omega_{m 2}^{2}},
$$

whereas the third dark line appears at the frequency of the probe resonance $\Delta_{p}=0$. This indicates that, in the presence of the strong coherent optical and microwave fields, the spectrum splits on four spectral components, caused by the dynamical Stark quartet effect in the strongly interactive energy levels of the atom.

The response of the absorption is shown in the Fig. 2 at the absence of the dephasing effect in the medium. In this case, we consider $\gamma_{1,2,3}=0.01 \gamma \approx 0$. However, to account 

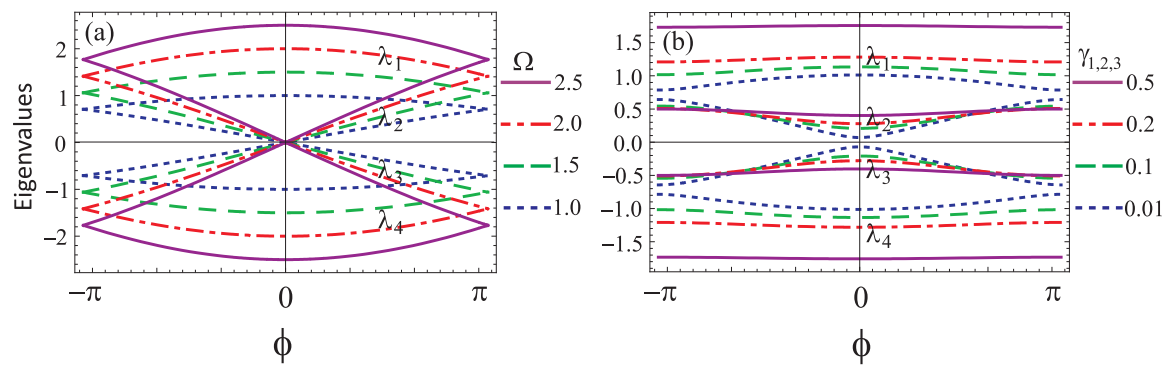

Figure 3. (Color online) The dressed energy eigenvalues $\lambda_{i}$ versus $\phi$ are shown in the panel (a) without the atomic dephasing, for $\Omega_{O 1}=\Omega_{O 2}=\Omega_{m 1}=\Omega_{m 2}=1 \gamma, 1.5 \gamma, 2 \gamma, 2.5 \gamma$. (b) Here the atomic dephasings $\gamma_{i=1,2,3}$ are considered to be in the rate $0.01 \gamma$ (blue dotted), $0.1 \gamma$ (dashed green), $0.2 \gamma$ (doted-dashed red), $0.5 \gamma$ (solid blue), and $\Omega_{O 1}=\Omega_{O 2}=\Omega_{m 1}=\Omega_{m 2}=1 \gamma$

for the influence of the atomic dephasing rates in the medium, various values higher than this dephasing rate in the medium are also considered here. Note, that the position of four spectral components depends on the relative phase of the coupling fields. At the relative phase $\pi / 2$, the four spectral components [with two of them being positive (negative)] are distinct with respect to the probe frequency detuning [see, e.g., Fig. 2(b)], under the condition of the absence of the dephasing effect in the medium. The spectrum remains identical, like an even function, by adjusting the relative phase at $3 \pi / 2$. The coherence evolution of the atomic system results in the four spectral components to be moved in a symmetric pattern with the reference to the probe frequency, tuning upon rotating the relative phase $\phi$ of the state vector of the atomic system into one complete cycle. At the relative phase $\pi$, each two spectral components on the positive (negative) side of the probe frequency detuning approach toward each other. As a result, at each side of the central dark line of the spectrum, two pairs of the spectral components converge due to the constructive quantum interference in the absorption spectrum. As a response, the two side dark lines collapse, closing the corresponding EIT windows; whereas the central EIT window survives for the propagation of the probe field [see Fig. 2(c)]. On the other side, by adjustment of the relative phase at 0 , the central two of the four components converge at the location of the probe resonance, when they approach towards each other. Therefore, the sides EIT windows remain opened in this case, whereas the central window closes for the probe transmission [see Fig. 2(a)].

\subsection{Coherent evolution of dynamical Stark quartet dressed state}

To get further insight into the phenomenon we study the system in the dressed states picture. In this case the Hamiltonian has the following form

$$
\begin{aligned}
\hat{H}_{I}= & \Delta_{p}|e\rangle\left\langle e\left|+\left(\Delta_{p}-\Delta_{01}\right)\right| g_{1}\right\rangle\left\langle g_{1}\right|+ \\
& \left(\Delta_{p}-\Delta_{s}\right)\left|g_{2}\right\rangle\left\langle g_{2}\left|+\left(\Delta_{p}-\Delta_{m 2}-\Delta_{s}\right)\right| g_{3}\right\rangle\left\langle g_{3}\right| \\
& -\frac{1}{2}\left[\Omega_{m 1}\left|g_{1}\right\rangle\left\langle g_{3}\left|-\Omega_{m 2}\right| g_{2}\right\rangle\left\langle g_{3}\left|-\Omega_{O 1}^{2}\right| e\right\rangle\left\langle g_{1}\right|\right. \\
& \left.-\Omega_{O 2}^{2}|e\rangle\left\langle g_{2}\left|+\Omega_{p}\right| e\right\rangle\langle g|\right]+ \text { H.c. }
\end{aligned}
$$


At the resonance condition $\Delta_{m 1}=\Delta_{m 2}=\Delta_{01}=\Delta_{s}=0$, the secular equation $\operatorname{Det}\left[\hat{H}_{I}-\lambda \hat{I}\right]=0$ yields

$$
\begin{aligned}
& 16 \lambda^{4}-4\left[\Omega_{O 1}^{2}+\Omega_{O 2}^{2}+\Omega_{m 1}^{2}+\Omega_{m 2}^{2}\right] \lambda^{2} \\
& \left.+\Omega_{O 1}^{2} \Omega_{m 2}^{2}+\Omega_{m 1}^{2} \Omega_{O 2}^{2}\right]-\Omega_{\text {eff }}^{4} \cos \phi=0 .
\end{aligned}
$$

This equation is identical to the expression of the parameter $D_{1}$, when $\lambda$ is replaced by $\Delta_{p}$. In this context, we obtain for the eigenvalues

$$
\begin{aligned}
& \lambda_{1,2}=\frac{1}{2 \sqrt{2}}[Q \pm A]^{\frac{1}{2}}, \quad \lambda_{3,4}=-\frac{1}{2 \sqrt{2}}[Q \mp A]^{\frac{1}{2}}, \quad A=\sqrt{Q^{2}-4 Z}, \\
& Z=\left[\Omega_{O 1}^{2} \Omega_{m 2}^{2}+\Omega_{O 2}^{2} \Omega_{m 1}^{2}\right]-\Omega_{\mathrm{eff}}^{4} \cos \phi, \quad Q=\Omega_{m 1}^{2}+\Omega_{m 2}^{2}+\Omega_{O 1}^{2}+\Omega_{O 2}^{2} .
\end{aligned}
$$

Note, that the dressed energy eigenvalues $\left\{\lambda_{i}\right\}$ [Eqs. (11)], associated with each strongly interactive energy level of the atom, depend on the relative phase $\phi$ of the coupling fields through the term $Z$. On the analysis of the eigenvalues we find that, similar to the four spectral components of the absorption spectrum, they manipulate their energy values with the reference to the energy of the corresponding bare-energy-level of the atom. The manipulation in the energy eigenvalues are in a regular and systematic pattern, when $\phi$ is subjected to one complete rotation in the Argand phase diagram of the state vector of this system. At equal intensities and relative phase $\pi / 2$ of the coupling fields, the four dressed eigenvalues, $\left\{\lambda_{i}\right\}$ , are equally separated from each other, as shown in the Fig. 3(a). At the evolution of the atomic system in the steady state limit, the eigenvalues $\lambda_{1}$ and $\lambda_{2}$, and, $\lambda_{3}$ and $\lambda_{4}$, coincide at either side of the bare-state, when $\phi$ is tuned into $\pi$. As a result, the four dressed states are coherently evolved into two dressed states: one up the bare-state, whereas the second down. Consequently, the quartet dressed state converts into the doublet dressed state. On the other hand, at the relative phase 0 , the eigenvalues $\lambda_{2}$ and $\lambda_{3}$ become equal at the probe resonance, located at the frequency position of the bare state. While the eigenvalues $\lambda_{1}$ and $\lambda_{4}$ take their position distinct: up and down with the respect to the central position of the former superimposed dressed states as the single central dressed state. In this case, the quartet dressed state of the atomic system is coherently converted into a triplet dressed state. We conclude that the quartet can be converted into the doublet or the triplet dressed states, based on the appropriate choices of the relative phases of the coupling fields.

Upon equally increase in the intensity of two optical fields and microwave fields, we note that the dressed states with their energy eigenvalues at relative phase: $0, \pi / 2$ and $\pi$, are separated from each other in each case. This means that the spacing among the dressed states are directly controlled by the Rabi frequencies $\Omega_{O 1, O 2, m 1, m 2}$ of the coupling fields. This behavior has a significant impact on the crossover from the EIT into the ATE in the medium.

\subsection{Competition of coherence controlled triple EIT with ATE}

Fig. 2 displays the absorption profile of the probe field under the influence of the dephasing reservoir. The larger the atomic dephasing rates in the medium (caused by the warm temperature medium consideration), the stronger the reduction in the destructive quantum interference. As a result, the dark line feature is gradually minimized in each case of the single, double, and triple EIT, as the dephasing decay rates are further increased in the medium. As a response, the EIT is gradually minimized in each case of the relative phase. Eventually, one observes the crossover of the EIT into the corresponding ATE in the medium. Note, that for sufficiently high dephasing decay rates in the medium, the appearance of the ATE is unnoticeable in the absorption spectrum. See, for example Fig. 2, the behavior of the EIT with the dephasing rate $\gamma_{1,2,3}=10 \gamma$ in each of the relative $\phi$. 
The threshold for the transition from the EIT into the ATE effect can be easily estimated by examining the dynamics, when the system is also subjected to the influence of the atomic dephasing reservoir [27]. To this end, we adopt the dressed state approach and calculate the energy eigenvalues, while considering the lossy Hamiltonian in the terms of dephasing decay rates $\gamma_{1,2,3}$ and radiative decay rate $\gamma$. Note, that the eigenvalues are identical to the roots of $\Delta_{p}$, when the condition $D_{1}=0$ holds [see Eq. (5)].

The dressed eigenvalues, evaluated numerically as a function of the relative phase $\phi$, are shown in Fig. 3(b). As compared to Fig. 3(a), the merging effect of the central two dressed states $\lambda_{2}$ and $\lambda_{3}$ at the relative phase 0 , and those of $\lambda_{1}, \lambda_{2}$, and $\lambda_{3}, \lambda_{4}$ (at the relative phase $\pm \pi$ ) is not exhibited by the system in the presence of the atomic dephasing decay rates in the medium. In this case, rather to be merged, each dressed state is more separated from its adjacent dressed state upon the increasing of the atom dephasing decay rates in the medium. In other words, the threshold of the atom dephasing corresponds to the suppression of the Rabi oscillations of the populations in the atom. As a result, the destructive quantum interference among the excitation probability amplitudes of the probe field is completely minimized for the threshold rate of the atomic dephasing in the medium. Therefore, the ATE corresponds to the non-perturbation regime of the coupling fields in the presence of the atomic dephasing. At the same time, the EIT is associated with the coherent Rabi oscillation of the atomic populations, induced by the coupling fields in the absence or relatively mild atomic dephasing rates in the medium.

However, even in the absence of the atomic dephasing decay rates in the medium, the EIT effect, in case of the relative phase, minimizes when the the intensity of the coupling field become sufficiently large. In this case, although the merging of the dressed states with the corresponding relative phase is exhibited, the normal dispersions and, subsequently the EIT effect, are reduced due to the relatively large separation of the dressed states of the strongly interactive energy level in atom, even though the merging phenomenon in this case. Therefore, the larger the intensity of the coupling fields, the smaller the normal and, subsequently, the milder the EIT effect in the probe field transmission. Eventually, the EIT transforms into the ATE. This behaviour is in accord with the results of [28], discussed, however, in the context of the triple EIT and switching effect in the medium.

\section{Conclusion}

We have investigated the coherence evolution of the response function of a five-level atomic system in the steady state limit, when the medium (in the presence of a weak probe field) is cyclically driven by two strong optical and microwave fields. The strong fields split each strongly interactive energy levels of the atom into a quartet to generate the triple EIT in the propagation of the probe field. We show that the quartet dressed atomic state is coherently evolved to convert into a triplet and a doublet dressed state upon tuning the relative phase of the coupling fields. In the presence of the atomic dephasing rate in a medium, we further show the evolution of the triple EIT into double and single ones, with the corresponding ATE in the probe transmission. The EIT phenomenon, in each case of the considered system, is, however, discerned from the ATE by using high threshold atom-field couplings or atomic dephasing rates in the medium. We show that the quartet dressed-state in the later case does not reveal the triplet and doublet dressed-state. On the other hand, each one dressed state of the doublet through the quartet dressed state adjacent to the others in the former case is well separated to switch the EIT into the ATE in the medium, although the conversion of the quartet dressed state into the triplet and doublet dressed states in this case exhibits. 


\section{References}

[1] U. Fano, Phys. Rev. 124, 1866 (1961)

[2] A. E. Miroshnichenko, S. Flach, and Y. S. Kivshar, Rev. Mod. Phys. 82, 2257 (2010).

C. Chin, R. Grimm, P. Julienne, and E. Tiesinga, Rev. Mod. Phys. 82, 1225 (2010)

[3] C. Ott et al., Science 340, 716 (2013)

[4] G. S. Agarwal, Quantum Optics (Cambridge University Press, 2013)

[5] D.-W. Wang, S.-Y. Zhu, J. Evers, and M. O. Scully, Phys. Rev. A 91, 011801 (2015). W. Chen, K. Zhang, D. S. Goldbaum, M. Bhattacharya, and P. Meystre, Phys. Rev. A 80, 011801 (2009)

[6] E. Arimondo, Prog. Opt. 5, 257 (1996)

[7] O. Kocharovskaya, Phys. Rep. 219, 175 (1992)

[8] L. V. Hau, S. E. Harris, Z. Dutton, and C. H. Behroozi, Nature (London) 397, 594 (1999)

[9] D. F. Phillips, A. Fleischhauer, A. Mair, R. L. Walsworth, and M. D. Lukin, Phys. Rev. Lett. 86, 783 (2001)

[10] J. P. Marangos, J. Mod. Opt. 45, 471 (1998)

[11] X. Xu, B. Sun, P. R. Berman, D. G. Steel, A. S. Bracker, D. Gammon, and L. J. Sham, Nature Phys. 4, 692 (2008)

[12] N. Liu, L. Langguth, T. Weiss, J. Kastel, M. Fleischhauer, T. Pfau, and H. Giessen, Nature Mater. 8, 758 (2009)

[13] Y. B. Martynov, R. G. Nazmitdinov, P. P. Gladyshev, and I. A. Tanachev, Journal of Physics: Conference Series 393, 012007 (2012)

[14] W. R. Kelly, Z. Dutton, J. Schlafer, B. Mookerji, T. A. Ohki, J. S. Kline, and D. P. Pappas, Phys. Rev. Lett. 104, 163601 (2010)

[15] N. Papasimakis, V. A. Fedotov, N. I. Zheludev, and S. L. Prosvirnin, Phys. Rev. Lett. 101, 253903 (2008)

[16] S. Zhang, D. A. Genov, Y. Wang, M. Liu, and X. Zhang, Phys. Rev. Lett. 101, 047401 (2008)

[17] A. H. Safavi-Naeini et al., Nature (London) 472, 69 (2011)

[18] W. E. Lamb and R. C. Retherford, Phys. Rev. 81, 222 (1951)

[19] C. L. G. Alzar, M.A.G. Martinez, and P. Nussenzveig, Am. J. Phys. 70, 37 (2002)

[20] K. P. Burnham and D. R. Anderson, Model Selection and Multimodel Inference (Springer-Verlag, New York, 2nd ed., 2002)

[21] M. Fleischhauer, A. Imamoglu, and J. P. Marangos, Rev. Mod. Phys. 77, 633 (2005)

[22] S. H. Autler and C. H. Townes, Phys. Rev. 100, 703 (1955)

[23] E. Saglamyurek et al., Nat. Phot. 12, 774 (2018)

[24] X-Q. Luo, Z-Z Li, J. Jing, W. Xiong, T-F Li and T-Y. S Sci. Rep. 8, 3107 (2018)

[25] F. Ghafoor, Laser Phys. 24, 035702 (2014). F. Ghafoor, Opt. Commun. 284, 1913 (2011). F. Ghafoor, Laser Phys. 24, 035702 (2014). F. Ghafoor and R. G. Nazmitdinov, J. Phys. B: At. Mol. Opt. Phys. 49, 175502 (2016)

[26] A. Imamoglu and S. E. Harris, Opt. Lett. 14, 1344 (1989)

[27] P. Anisimov and O. Kocharovskaya, J. Mod. Opt. 55, 3159 (2008)

[28] T. Y. Abi-Salloum, Phys. Rev. A 81, 053836 (2010)

[29] P. M. Anisimov, J. P. Dowling, and B. C. Sanders, Phys. Rev. Lett. 107, 163604 (2011)

[30] L. Giner et al., Phys. Rev. A 87, 013823 (2013)

[31] C. Zhu, C. Tan, and G. Huang, Phys. Rev. A 87, 043813 (2013)

[32] G. Tiaz, A. E. Shahalyev A. Ashiq and F. Ghafoor, submitted to IEEE: Quantum Electronics (2019) 